DOI: 10.20472/IAC.2018.043.017

\author{
R. BOYD JOHNSON \\ Indiana Wesleyan University, United States \\ DIANA MIRZA-GRISCO \\ Cultural Intelligence in Eastern Europe Project, Romania
}

\title{
THE ADAPTATION OF THE CULTURAL INTELLIGENCE SCALE IN CENTRAL AND EASTERN EUROPE
}

\begin{abstract}
:
The cross-cultural adaptation of quantitative research instruments represents a challenging process that requires methodological exactness. A good example is the Cultural Intelligence Scale (CQS). Even though the CQS has been used in 98 countries, there are few studies which examine the transferability of cultural intelligence concepts in former socialist countries (Barnes, Johnson, Buko, 2010). In cross-cultural research it is essential to take into consideration how other cultures interpret and view concepts and questions, since it has a direct impact on the answers of respondents and empirical findings (Bostjancic, Beljak, Johnson, 2016).

This presentation will report on an empirical example of cross-cultural adaptation of research instruments, carried out by a global team from the USA and Europe. It is based on the adaptation of the Cultural Intelligence Scale (CQS), which measures the capability of a person to relate and work effectively in culturally diverse situations. The CQS contains four factors: metacognitive, cognitive, motivational and behavioral. The authors discuss their approach of adapting the cultural intelligence scale in four countries from Central and Eastern Europe, taking into consideration different cultural nuances across geographical lines: Ukraine, Republic of Moldova (two former soviet republics), Slovenia and Serbia (two former Yugoslav repubics). In each of the four countries the same methodology was applied with the intent to adjust the CQ scale and then measure cultural intelligence nation-wide in representative studies (over 1000 respondents). The authors discuss the three stages used to adapt the CQ instrument: first, using forward and back translation, second, focus groups with two different types of local populations in each country, and third, pilot studies with university students. Throughout, the results were assessed together with local experts. The statistical analyses of the adapted scale (in each country) demonstrated very high reliability and that the factor structure fits the four subscales. This research offers new insights for CQ competency application in intercultural management and leadership studies, and in social sciences for both academics and practitioners.
\end{abstract}

\section{Keywords:}

cross-cultural instrument adaptation, cultural intelligence, focus group, transferability. 